\title{
Electromyographic evaluation of selected muscles during active hip extension in men treated McKenzie method
}

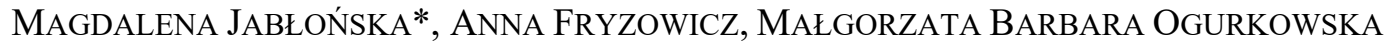 \\ Department of Biomechanics, Poznan University of Physical Education, Poznań, Poland.
}

\begin{abstract}
Purpose: The aim of this study was to evaluate objective results of short-term treatment with the use of electromyography and to determine changes in muscle recruitment during the prone hip extension test in individuals with chronic low back pain who underwent the McKenzie treatment. Methods: The studied group consisted of 17 male office workers aged 33-55 who underwent two experimental sessions comprising of 3 measurements and the McKenzie method of mechanical diagnosis and therapy. Electromyographic examination of selected muscles was performed during the test in order to assess changes in their activity before and after the McKenzie method and the Visual Analogue Scale was used to assess the level of pain. Results: There was a tendency towards significance at the beginning of activation between the first and second test for the left erector spinae muscle $(p=0.0684)$. In $18 \%$ of patients, the onset activation time decreased, which is indicative of correct direction of changes. No statistically significant changes were observed in the remaining muscles. Statistical significance ( $p=0.0131$ ) was observed between the first and third test for the left erector spinae muscle. A decrease in the onset activation time was observed in $75 \%$ of the subjects. Effectiveness increased by as much as $57 \%$. The level of pain decreased in the third test compared to the first one $(p=0.0240)$. However, the change in pain sensations between the first and second test was not statistically significant. Conclusions: The study showed objective changes in the activation sequence of selected muscles and subjective changes in pain following short term McKenzie therapy.
\end{abstract}

Key words: low back pain, sEMG, movement pattern, prone hip extension

\section{Introduction}

Nowadays, back pain constitutes a serious social and economic problem. It affects about $80 \%$ of population at various stages of life and it is the most frequent cause of disability among adults. Epidemiological data show that $42-75 \%$ of people who experienced the first episode of low back pain (LBP) also experience relapses [1]. The McKenzie method, whose effectiveness exceeds that of other rehabilitation interventions, is one of the ways to effectively combat chronic sacral pain. The McKenzie method is a mechanical diagnosis and therapy of spine and limb pain syndromes based on the knowledge of pain patterns and the analysis of changing symptoms in interview and physical examination. Mechanical diagnosis is based on the evaluation of symptomatic reactions during performance of repeated movements. The patient's task is to determine the symptoms occurring before, during and after the movements, and then to determine whether the symptoms change during repeated movement and afterwards. Evaluation of the reactions consists in determining peripheralisation and centralisation of symptoms [16].

One of the best studies evaluating the effectiveness of the McKenzie method was carried out by Long et al. [19]. The study assessed the importance of the exercise performed by the patient. It was shown that in a group of patients who performed the exercise selected in accordance with the directional preference shown in the diagnostic process, there was a significant improvement or complete relief of pain in $95 \%$ of the patients. In patients who performed exercises

\footnotetext{
* Corresponding author: Magdalena Jabłońska, Department of Biomechanics, Poznan University of Physical Education, Królowej Jadwigi 27/39, 61-871 Poznań, Poland. E-mail: magdalena.zygmanska@wp.pl

Received: February 25th, 2020

Accepted for publication: May 13th, 2020
} 
in the opposite direction, there was an improvement in $23 \%$ of patients.

However, literature review indicates a subjective evaluation of the effectiveness of the McKenzie method with patients noticing a reduction in pain or disability after the McKenzie method of treatment [6].

Available studies indicate possible causes of LBP. First of all, the presence of changes in the muscle system is mentioned as the main cause of abnormalities. Spine stability disorders are caused by distorted antagonist/agonist balance or low level of muscle activation [10]. Moreover, in the Tikkanen et al.'s study [25], a lower muscle activity was observed in men, compared to women, during normal daily life. Therefore, the task of the muscle system is to control movement and stabilize spinal structures, thereby protecting against overloading during physical activity. Muscles protect the spine from damage, but, as a result of prolonged overloading, they become inefficient and the loads will be transferred to the passive stabilization system (ligaments, capsules of intervertebral joints). If the movement which causes overloading is repeated, it causes damage to the intervertebral disc and morphological changes in the vertebral bodies [21].

Overloading of the spine may occur during everyday activities, professional work and competitive sports [21]. Office workers are likely to spend long hours in a forced seated position in front of a computer. It was proven that the seated position alone is not a factor in the development of back pain, but working in uncomfortable positions for more than half a day's work is. Moreover, the time spent working in the seated position affects the intensity of LBP among blue-collar workers, as well as in white-collar workers. O'Sullivan et al. [22] determined the relationship between body posture, muscle endurance and pain in industrial workers complaining of back pain associated with the bending of the lumbar spine. It was proven that passive, slump sitting postures lead to dysfunction of the muscles stabilizing the spine, decrease of muscle endurance, increase of pelvic frontal tilt in the seated position and spinal flexion at the limits of the mobility range during "normal" sitting. This alignment of the spine may lead to increased loads on the passive system of stabilizers and decreased activity of active stabilizers of the spine, i.e., the muscle system. Permanent and cyclically repeated abnormal motor activity and sitting in an abnormal position for prolonged periods of time may contribute to changes in muscle tissue and its dysfunctions, manifested by a changed movement pattern, pain or, eventually, motor disorders [22]. Studies indicate that LBP is associated with muscular imbalance and a change in the pattern of lumbo-pelvic muscle activation during various tasks Hungerford et al. [12] described a delay in electromyographic activation in patients with LBP, compared to healthy subjects, during a test which involved hip flexion in the standing position. This shows a change in the strategy of stabilization of the sacroiliac joints, which may result in disorders in load transfer through the pelvis [12]. Leinonen et al. [17] examined patients with LBP. During the test of torso flexion and extension, changes in the activation of individual muscles were also observed in the sagittal plane. Reduced gluteus maximus muscle (GM) activity was observed, which helped formulate guidelines in terms of rehabilitation of these patients [17].

Motor patterns in individuals with back pain can be evaluated in clinical tests. One of them is the prone hip extension (PHE) test, developed by Janda. It is widely used to measure the lumbo-pelvic movement pattern. The test consists in lifting the lower limb - straight knee joint - in the prone position. Janda [14] was the first to describe muscle recruitment dysfunction as a pathology. He examined the sequencing between synergistic muscles during selected functional movements. Comparison of muscle activity sequences in people experiencing pain and health problems made it possible to define specific recruitment patterns. During the above mentioned test, an imbalance of muscles and changed recruitment of lumbo-pelvic muscles in patients with back pain were observed [14]. It is assumed that the change in lumbosacral muscle activation contributes to the destructive effect on the system of stabilizers. Studies show that in workers with back pain, the lumbar muscles activate too quickly in comparison to healthy people [26].

Despite many studies evaluating the efficacy of the McKenzie method in individuals with spinal pain syndrome, there is still some controversy in terms of diagnosis and treatment of this group of patients, and studies conducted to evaluate treatment results are mainly based on subjective scales and questionnaires. For this reason, it was decided to evaluate the results of treatment of patients with chronic lumbar pain on the basis of objective methods of movement analysis.

The aim of this study was to evaluate objective results of short-term treatment with the use of surface electromyography (sEMG) and to determine changes in muscle recruitment during the PHE test in individuals with chronic lumbar and sacral pain who underwent the McKenzie treatment.

Moreover, differences between subjective and objective results of short-term McKenzie therapy were compared and evaluated. 


\section{Materials and methods}

\subsection{Ethics statement}

The study was approved by the Bioethics Committee of the Poznan University of Medical Sciences. All subjects expressed written informed consent to participate in the study. All procedures were conducted according to the Declaration of Helsinki.

\subsection{Participants}

The studied group comprised 17 men (average age: 41.2 years, standard deviation (SD): 6.89; average body mass index: $27.39 \mathrm{~kg} / \mathrm{m}^{2}$, SD 3.86; average body weight: $87.9 \mathrm{~kg}$, SD 11.7; average body height $178.9 \mathrm{~cm}$, SD 5.9) with chronic non-specific lumbar and sacral pain syndrome, working in the seated position in front of a computer (average work experience 13.3 years, SD 6.59).

Patients were preliminarily classified with the use of a lumbar spine examination protocol according to the McKenzie method. Moreover, anthropometric data, information on the job seniority and nature of work, activities and tasks performed outside of work or knowledge of ergonomic principles were collected. Respondents indicated the frequency of pain, functional limitations, daily variability of pain and the influence of body positions on pain.

The criteria for inclusion in further tests included: the presence of features characteristic of the derangement syndrome, in which pain is caused by mutual disturbance of structures in the motor segment (e.g., displacement of the nucleus pulposus leading to annulus fibrosus damage and even pressure on nervous structures). Centralisation and peripheralisation as well as directional preference were characteristic only for this spinal pain syndrome. In addition, there was a feeling of "movement blockage", pain in the lumbo-sacral region, buttocks or thighs, history of similar pain episodes in the past, and a changing picture of symptoms throughout the day and over time. The following exclusion criteria were defined: history of lumbar spine surgery, diagnosed spondylolisthesis, history of hip, knee and ankle joint pain, female sex.

\subsection{Experimental procedures}

In the first stage of the study, all patients $(n=44)$ underwent an examination based on the McKenzie protocol (Fig. 1). During the test, the therapist's task was to see whether directional preference and centralisation could be determined. Patients in whom directional preference and centralisation were not defined were excluded from the study.
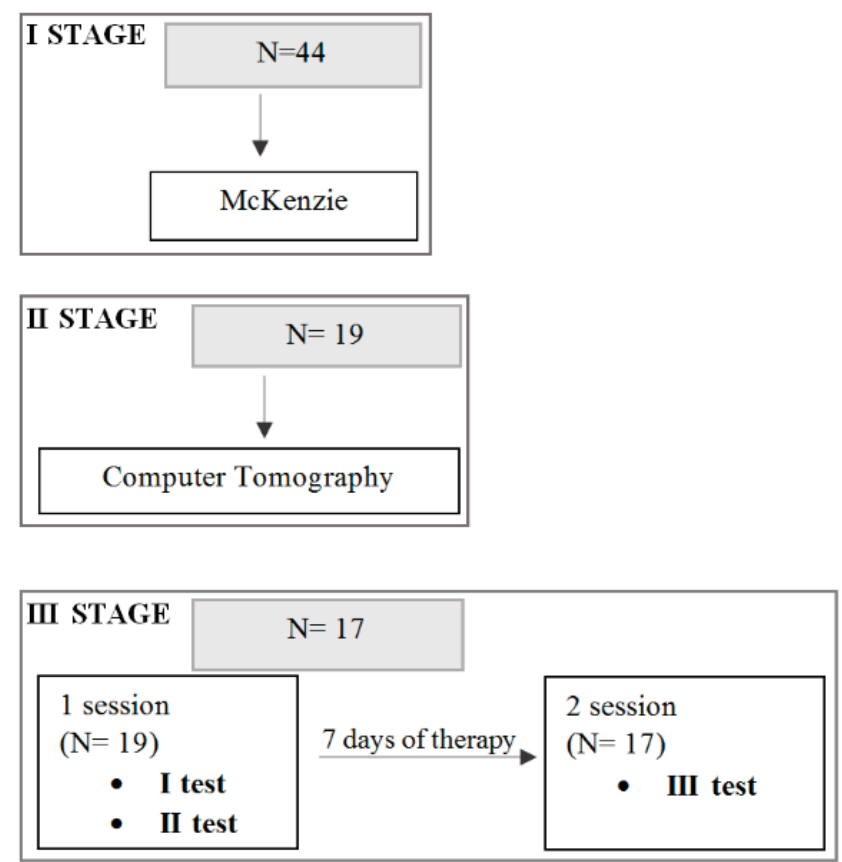

Fig. 1. Diagram describing the number of patients at particular stages of the study

In the second stage of the study, the patients ( $n=19)$ underwent Computed Tomography scan of the lumbar spine (Fig. 1). The examination was carried out in an independent diagnostic imaging laboratory. Descriptions of the examination results were prepared by a radiologist. All examined individuals were diagnosed with overload degenerative changes in the form of protrusion of intervertebral discs located centrally, bi-sided in 17 individuals; centrally, left-sided or right-sided in the remaining individuals; 13 individuals were diagnosed with narrowing of intervertebral space. The exclusion criteria at this stage were: neoplastic disease, previous fractures of the spine.

The third stage of the study was divided into two sessions. In the first session 19 patients participated and it included: warm-up, I evaluation of pain according to the Visual Analogue Scale (VAS), I sEMG measurement during PHE test and the McKenzie method therapy. Then, after an hour break, the following were performed: II pain evaluation according to the VAS scale, and II sEMG measurement during PHE test. The second session took place after 7-day therapy according to the McKenzie method, per- 
formed individually at home and at work. Two patients decided not to continue after the first session $(n=17)$. During the second session, patients underwent III pain assessment according to the VAS scale and III sEMG measurement during PHE test (Fig. 2).

\begin{tabular}{|l|l|}
\hline \multicolumn{1}{|c|}{ Session 1 } & \multicolumn{1}{|c|}{ Session 2 } \\
\hline - Warm-up & - Warm-up \\
- 1 VAS scale & - 3 VAS scale \\
- 1 sEMG measurement & -3 sEMG measurement \\
- McKenzie therapy & \\
- 2 VAS scale & \\
- 2 sEMG measurement & \\
- Exercise instructions & \\
\hline
\end{tabular}

Fig. 2. Schedule of the third stage of the study

\section{4. sEMG measurement}

PHE test was used to as a tool to assess the effectiveness of short-term therapy according to the McKenzie method. During the PHE test, sEMG measurements were performed with the use of the 16-channel telemetric sEMG TeleMyo 2400T G2 system (Noraxon, USA). The sEMG signal was sampled at $1000 \mathrm{~Hz}$ and then filtered (bandwidth: 10-500 Hz). Recruitment of 4 selected lumbo-pelvic muscles was analysed: left and right erector spinae muscles (LES and RES), right gluteus maximus muscle and right biceps femoris muscle (BF). The subjects were asked to lie down in the prone position on the couch with upper extremities resting along the body. Next, the patient's skin was shaved and cleaned. According to SENIAM recommendations, disposable, self-adhesive $\mathrm{Ag} / \mathrm{AgCl}$ electrodes (SORIMEX, Poland, $1 \mathrm{~cm}$ in diameter) were placed in a bipolar configuration, parallel to the muscle fibres. The electrodes were stuck by a physiotherapist. In order to collect sEMG signals, the electrodes were placed as follows: for RES and LES on both sides at least $2 \mathrm{~cm}$ from the spinous processes of the L3 vertebra, for GM - in the middle of the line connecting S2 with the greater trochanter, for $\mathrm{BF}$ - laterally in the middle of the line between the buttock fold and the hollow of the knee [8]. When requested, the patient did prone hip extensions, approx. angle of 20-30 degrees. The patient maintained this position for three seconds and then returned to the initial position. Five PHE tests were carried out for the right lower limb.

\section{5. sEMG signal processing}

Three correctly recorded results were selected for analysis. Signal processing was performed with the MyoResearch XP Master Edition software (Noraxon, USA). Artefacts and noise were visually inspected. The sEMG signal was full-wave rectified and smoothed using a root mean square algorithm with a 50 millisecond window. The sequence and time of activation of ES, GM and BF muscles were measured. It was assumed that the onset of muscle activation was when the sEMG signal was maintained at the level over 3 standard deviations from the average value of the signal recorded at rest in the prone position for a minimum period of 0.3 seconds. The activation time of the BF muscle was set as the reference point, for which the relative activation time was marked as " 0 ". Next, the relative activation time for each of the examined muscles was calculated according to the following formula:

\section{Relative activation time of muscle $X$ $=$ absolute activation time of $B F$$$
\text { - absolute activation time of muscle } X
$$

The result for muscles that activated before $\mathrm{BF}$ is positive, while in the case of muscles that activated after $\mathrm{BF}$ the result is negative.

The reference group was established on the basis of standards for the correct muscle activation sequence during the PHE test according to Janda [14]. Janda suggests that the regular activation pattern during hip prone extension is as follows: hamstrings, followed by GM, followed by contralateral ES, followed by ipsilateral ES.

\subsection{McKenzie method therapy}

The therapy during the first session was based on the McKenzie method of Mechanical Diagnosis and Therapy. It consisted of performing a specific movement of the torso during which symptoms were centralised or eliminated. Static and dynamic procedures were applied. The first procedure consisted in assuming a static forearm-supported prone position for five minutes. Then the dynamic procedure followed. The hyperextension pattern, which consisted in active torso hyperextension in the prone position, was applied again. The therapy was broken down into 4 series, 10 to 15 repetitions each.

At the end of the first session, the subjects received instructions with the schedule of the self- 
therapy. The duration of the therapy was 7 days. It was recommended to perform the static and dynamic procedure cyclically, every 2 or 3 hours, interrupting the work / seated position for a few minutes. In addition, in case of pain, the use of self-therapy on an ad hoc basis was also recommended. All patients were informed about the phenomenon of centralisation and peripheralisation and the necessity to report intensification of pain symptoms by phone.

\subsection{VAS scale}

The VAS scale was used at each stage of the study. The VAS scale is a visual-analogue scale used to determine the intensity of pain sensations. No pain corresponds to 0 , and unbearable pain, the largest that a patient can imagine, corresponds to 10 . The patient is recommended to determine the intensity of pain on a scale drawn on a piece of paper [20].

\subsection{Statistical analysis}

The aim of the analysis was to determine whether a short-term McKenzie intervention affects the order of muscle activation during the PHE test and the level of pain experienced by patients. The mean time (from three tests) of muscle activation onset was calculated. This constituted the basis for determining the order of muscle recruitment in all patients. In addition, changes in pain sensations were determined on the three test dates with the use of the VAS scale.

\subsection{Statistical methods}

Results of quantitative variables used in the study were presented as mean values, standard deviations (SD), while qualitative variables were presented as percentage and number. Normality of distribution was examined and conformity assessment was performed with the Shapiro-Wilk test. Critical significance level was assumed at $p=0.05$. The analysis of significance of differences between the two tests over time was carried out with the Wilcoxon's test. Correlations between the pain scale and muscle activation sequence were performed using Spearman's rank correlation coefficient test.

To describe differences related to two averages, effect sizes $(d)$ were calculated as the difference between means divided by the pooled standard deviation. Using Cohen's criteria [7], an effect size $\geq 0.20$ and $<0.50$ was considered small, $\geq 0.50$ and $<0.80$ medium, and $\geq 0.80$ large.

Statistical analysis of the results was performed with the use of the STATISTICA 10 statistical package (StatSoft Inc.).

\section{Results}

Table 1 shows the activation onset time of selected muscles on three test dates and the sequence of recruitment in all the subjects. The muscle activation sequence during the PHE test was determined with reference to the $\mathrm{BF}$ muscle, which should be the first to activate.

The BF muscle, which should be the first to activate in all the tests, showed a significant improvement between the first and the third test. In the first study, it was the first to activate in 5/17 (29\%) of the subjects, a deterioration was observed in the second test (4/17 participants, 24\%), while the third test showed an improvement, with BF the first to activate in $8 / 17(47 \%)$ of the subjects (Fig. 3). What is also worth noting, in the first test the BF muscle was the last to activate in 8/17 (47\%) individuals. Faster activation was observed after the therapy, as in the second test it was the last to activate in 6/17 (35\%) of the subjects and in the third test only in 4/17 (24\%) (Table 1).

The GM muscle should be the second to activate. In the first test it was activated second in $1 / 17(6 \%)$ subjects, in the second test: in $2 / 17(12 \%)$ and in the third test: $3 / 17(18 \%)$ of the subjects (Table 1$)$.

The third muscle is LES, which was the third to activate in $7 / 17(41 \%)$ subjects in the first test. The activity of the LES muscle deteriorated, which was observed in the second and third test, where the muscle was the third to activate only in $3 / 17(18 \%)$ of the subjects. However, it is worth noting that after the therapy, LES became increasingly often the fourth to activate. In the first test it was the fourth to activate in $2 / 17(12 \%)$ of the subjects, in the second test - in $4 / 17(24 \%)$ of the subjects, and in the third test - in 5/17 (29\%) of the subjects (Table 1).

The last muscle should be the RES muscle, which was the fourth to activate in all the tests in $3 / 17(18 \%)$ of the subjects (Table 1). The RES muscle was also the first to activate in the first test in as many as $8 / 17$ $(47 \%)$ of the subjects. Following the first therapy, in the second test, a decrease to $6 / 17(35 \%)$ was observed, and after another therapeutic intervention, in the third test, the RES muscle was the first to activate only in $4 / 17$ (24\%) of the subjects (Fig. 3 ). 
Table 1. Average time (ms) of the onset of activation of selected muscles

in relation to Biceps Femoris (BF) firing time in all patients in three tests

\begin{tabular}{|c|c|c|c|c|c|c|c|c|c|c|c|c|c|}
\hline \multirow{3}{*}{$\begin{array}{l}\text { Number } \\
\text { of patients }\end{array}$} & \multicolumn{13}{|c|}{ Average time of the onset activation of selected muscles $(\mathrm{ms})$ in three consecutive tests (I-III) } \\
\hline & \multirow{2}{*}{\begin{tabular}{|c|}
$\mathrm{BF}(1)$ \\
I-III tests
\end{tabular}} & \multicolumn{3}{|c|}{ GM (2) } & \multicolumn{3}{|c|}{ LES (3) } & \multicolumn{3}{|c|}{ RES (4) } & \multicolumn{3}{|c|}{ ORDER (1-4) } \\
\hline & & I test & II test & III test & I test & II test & III test & I test & II test & III test & I test & II test & III test \\
\hline 1 & 0 & -21.0 & 17.3 & -14.7 & -5.3 & 19.3 & -14.3 & -19.3 & 18.0 & 64.9 & 1342 & 3421 & 4132 \\
\hline 2 & 0 & -11.0 & -11.3 & -5.7 & -7.3 & -4.0 & -26.7 & 11.7 & -2.3 & -21.2 & 4132 & 1432 & 1243 \\
\hline 3 & 0 & -35.0 & -16.0 & -15.3 & -34.7 & 46.3 & -17.7 & 3.3 & -58.3 & -9.7 & 4132 & 3124 & 1423 \\
\hline 4 & 0 & -29.7 & 1.7 & 1.0 & -6.0 & -5.0 & -1.3 & 112.0 & -2.7 & -79.3 & 4132 & 2143 & 2134 \\
\hline 5 & 0 & -10.3 & -10.0 & -30.3 & -12.3 & -41.3 & -19.7 & -10.4 & -29.3 & 0.1 & 1243 & 1243 & 1432 \\
\hline 6 & 0 & -56.3 & -54.3 & -79.3 & -76.0 & -37.0 & -69.7 & -56.0 & -45.0 & -71.3 & 1423 & 1342 & 1342 \\
\hline 7 & 0 & -8.3 & -6.3 & -5.0 & 2.3 & 5.0 & 2.3 & 86.8 & 263.5 & 1.3 & 4312 & 4312 & 3412 \\
\hline 8 & 0 & -30.3 & -42.7 & 6.7 & -28.3 & -24.7 & -32.7 & -19.7 & 38.7 & -23.3 & 1432 & 4132 & 2143 \\
\hline 9 & 0 & -5.0 & -3.0 & -7.0 & 19.7 & 3.0 & 8.0 & 23.7 & 7.3 & 1.0 & 4312 & 4312 & 3412 \\
\hline 10 & 0 & -38.7 & -47.3 & -48.2 & -32.7 & 15.7 & -74.5 & -1.7 & 109.2 & -37.2 & 1432 & 4312 & 1423 \\
\hline 11 & 0 & -4.7 & -27.3 & -15.0 & 3.7 & 10.7 & -3.0 & -75.7 & -5.8 & -51.0 & 3124 & 3142 & 1324 \\
\hline 12 & 0 & -0.7 & -6.7 & -8.0 & 137.8 & 4.7 & -7.0 & 33.3 & 0.1 & -16.0 & 3412 & 3412 & 1324 \\
\hline 13 & 0 & -4.0 & 5.0 & -6.3 & 12.0 & 21.3 & 0.1 & -8.7 & 20.7 & 12.3 & 3124 & 3421 & 4312 \\
\hline 14 & 0 & 25.2 & -28.7 & -1.0 & -37.8 & -37.3 & -51.7 & -70.5 & -54.3 & 0.3 & 2134 & 1234 & 4123 \\
\hline 15 & 0 & -4.7 & -7.7 & -8.0 & -174.2 & -169.0 & -445.0 & 259.3 & 265.0 & -76.3 & 4123 & 4123 & 1243 \\
\hline 16 & 0 & -23.1 & -6.7 & 70.4 & -18.4 & 2.7 & -57.7 & 3.0 & 106.1 & -33.0 & 4132 & 4312 & 2143 \\
\hline 17 & 0 & -7.0 & -0.7 & 13.3 & -7.3 & 39.0 & -2.7 & 1.0 & 17.7 & 15.0 & 4123 & 3412 & 4213 \\
\hline Mean & 0 & -15.56 & -14.39 & -8.96 & -15.58 & -8.96 & -47.84 & 16.01 & 38.15 & -19.02 & - & - & - \\
\hline S.D & 0 & 18.73 & 19.47 & 29.89 & 59.53 & 48.26 & 105.58 & 78.89 & 96.82 & 37.12 & - & - & - \\
\hline
\end{tabular}

BF - Biceps Femoris, GM - Gluteus Maximus, LES - left Erector Spinae, RES - right Erector Spinae, ms - milliseconds, SD - Standard Deviation.

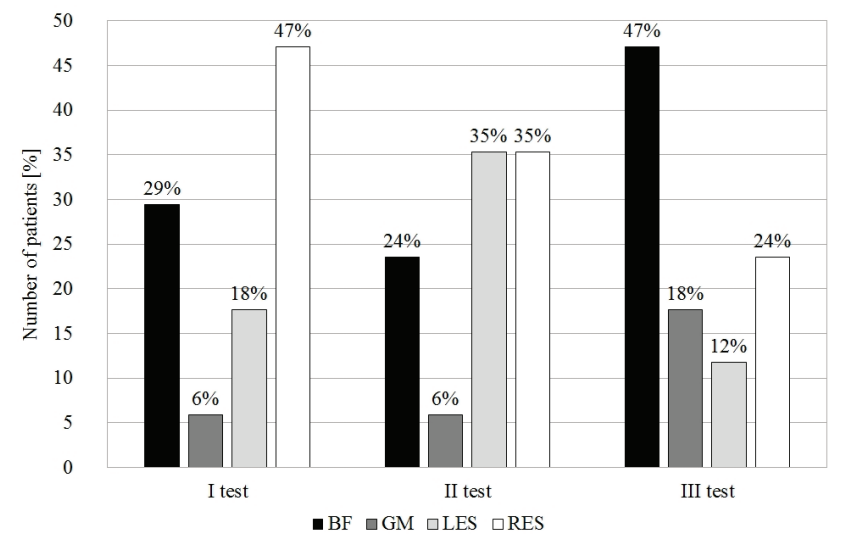

Fig. 3. Comparison of muscles which were the first to activate in the three tests

Comparison of muscle recruitment between the tests showed that there was a tendency for relevance at the onset of activation between the first and second test for the LES muscle ( $p=0.0684)$. In 14/17 (82\%) of the subjects an increase in the onset activation time of this muscle was observed. Therefore, in 3/17 (18\%) of patients the onset activation time decreased, which is indicative of correct direction of changes. Statistical significance $(p=0.0131)$ between the tests of muscle recruitment time occurred between the first and third test for the LES muscle. A decrease in activation time was observed in $13 / 17(75 \%)$ of the subjects. Effec- tiveness increased by as much as $57 \%$. No statistically significant changes were observed in the remaining muscles.

The level of pain assessed according to the VAS scale decreased in the third test compared to the first one $(p=0.0240)$. However, the change in pain sensations between the first and second test was not statistically significant. Comparison of pain level in the three tests is presented in Fig. 4.

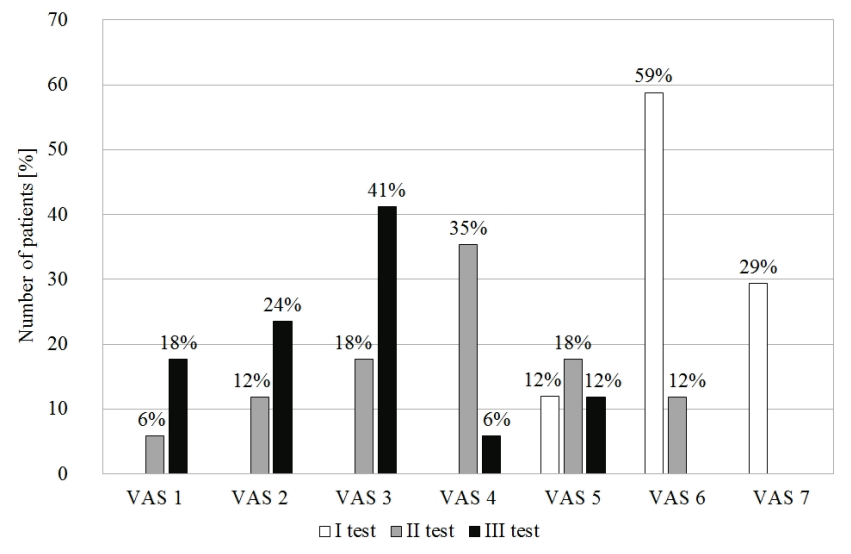

Fig. 4. Comparison of pain level in the three tests

In the first test, the average pain level on the 10-degree VAS scale was 6 . After the first therapy, the average pain level decreased to 4 , as reported by $94 \%$ 
of the subjects. This change was statistically significantly varied and the effect of the change was high $(p=0.0005, d=2.2)$. After the second therapy, in the third test, the level of pain decreased on average by 3 points on the VAS scale, reaching the average level of 3. A decrease on the VAS scale was observed in all the subjects. This change was statistically highly significant and the effect was even higher than between the first and second test ( $p=0.0003, d=3.6$ ).

Comparing the decrease in pain sensations between the first and the second test, it was noted that $7 / 17$ (41\%) of the subjects experienced a decrease in pain by 2 points on the VAS scale, $3 / 17(18 \%)$ of the subjects - by 1 point, and pain decrease by 3,4 and 5 points was experienced by $2 / 17(12 \%)$ of the subjects each. In 1/17 (6\%) of the individuals the level of pain increased by 1 point on the VAS scale. Comparison of the first test with the third test showed that the level of pain decreased in all individuals. In 5/17 $(29 \%)$ of the subjects pain decreased by 3 points; pain reduction by 2,4 and 5 points on the VAS scale was reported in equal number of instances in three groups of $4 / 17(24 \%)$ of the subjects. It should be added that for 2 individuals the change between the first and second test was the same as between the first and the third test.

There was no correlation between the objective results obtained in the test of muscle activation sequence and subjective results described with the use of the VAS scale $(p>0.05)$.

\section{Discussion}

This study assessed the change in the activation sequence of selected torso and lower limb muscles during the PHE test in individuals with chronic lumbar spine pain undergoing the McKenzie therapy and its influence on pain sensations. The results of the first test indicate an abnormal muscle stimulation sequence in relation to normative data on activation sequences of lower limb and paraspinal muscles [23]. Suehiro et al. [24] found that people with chronic sacral pain apply a changed and inappropriate pattern of recruiting torso muscles.

After short-term therapy, changes in the activation sequence of selected muscles were observed during the PHE test. A considerable improvement in the sequence was observed in the BF muscle in the third test, as an increasing number of individuals was activating it in the correct order. GM muscles also showed an improvement and came second in the muscle activation sequence in the test, both in the second test prior to the first therapy and in the third test after a week of self-therapy. However, the activity of this muscle was not consistent with quite significant differences in the onset of activation in the tests carried out. It was observed that the activity of paraspinal muscles changes quite significantly after therapeutic interventions. These muscles should be activated last and this change was observed in the second and third test.

$\mathrm{BF}$ also showed an improvement in that in the first test it was usually the last to activate instead of first. After the first as well as the second therapy there was an improvement in the activation sequence of this muscle. It is also worth noting that as a result of the therapy paraspinal muscles became active increasingly later, which means that they did not activate before the muscles which should initiate the hip extension movement. Therefore, there were changes in the sequence of the onset of activation of selected muscles with a general tendency towards improvement following short-term therapeutic interventions according to the McKenzie method.

The tests indicate that the paraspinal muscles (RES, LES) activate much too quickly during the PHE test, which may be indicative of excessive strain on spinal structures resulting from their hyperactivity. The study material showed features characteristic of changes in the intervertebral discs, which is indicative of abnormal strain on the lumbar spine. This contributes to further weakening of passive stabilizers. Therefore, stabilization control is mainly taken over by the system of active stabilizers, thereby demonstrating excessive activity [21]. Studies show that in workers with back pain lumbar muscles activate too quickly in comparison to healthy people [9], which is confirmed in this paper.

The results confirm Danneels et al. thesis [9] that the electromyographic activity of selected muscles in patients with chronic lumbar pain differs from healthy individuals performing the same motor tasks. Other authors also obtained similar results in their studies. In addition, there was a difference between symptomatic and asymptomatic side in individuals with sacroiliac joint pain. Similarly, studies conducted by Ji-Won Kim et al. [15] report increased normalized amplitudes of electromyographic signal in patients with back pain.

For the lower right extremity, a tendency towards improvement of the relative activation time of the GM and BF muscle during the PHE test was observed by comparing three measurements. This was also confirmed by the Chance-Larsen et al. study [5], in which an improvement in the activation sequence after a short exercise intervention was observed. 
It was observed that the muscle activation pattern also differs between healthy and injured persons [4]. In addition, the altered muscle activation appears not only on the side of the injured lower limb, but also the opposite side. The delay in the onset of GM activation constituted a significant difference between the studied groups. Bullock-Saxton [4] found a statistically significant delay in GM activation during the PHE test in individuals after an ankle joint sprain episode. There are studies that show the effect of nociception on muscle activity. Results of studies performed by Bruno and Bagust [2] indicate delays in GM activation during the PHE test in patients with chronic LBP, which is indicative of a change in the correct movement pattern observed in this study.

Lewis and Sahrmann [18] confirm the thesis concerning the constancy of the order of muscle activation despite individual variability during the performance of specific motor tasks in healthy individuals. In order to confirm the programmed movement pattern, several other studies were carried out with a different type of functional movements. A study by Friedli et al. [11] also showed the correct, predicted order of recruitment of muscles of the lower limbs and trunk when performing specific motor tasks based on the example of the elbow joint.

In this study, the activation sequence of the GM evaluated during the lower right limb test was analysed. Considering its order of activation during PHE in the first and second measurement, the highest value of delay in activation in the established norm, i.e., as the second to activate, was observed. In the first test it most frequently initiated the movement, but after a short therapy it was already one of the last muscles to be activated. Bruno et al. [3] also noticed a change in GM activity. It was considered that the PHE test could be a potential tool for assessing whether GM is a "weak link" in motor control strategies. In addition, the PHE test can be used as an indicator to recommend therapy that restores the "normal" pattern of activation sequence of this muscle [3].

However, there are studies that showed delays in GM activation in individuals with lumbar pain as well as in healthy individuals [3]. Questioning the hypothesis of predictable order of muscle activation was related to the results of electromyographic activity assessment studies, which failed to distinguish between people with and without sacral pain [3]. In this paper, GM onset activation sequence of may be considered inconsistent.

The results of subjective tests, conducted with the use of the VAS scale, suggest very good analgesic effects of the McKenzie method. Authors of other studies also confirm a significant improvement in the evaluation of pain. Ibrahimaj et al. [13] stated that as early as after 15 days of therapy the pain level decreased from the average value of 9 to 2 on the VAS scale.

\section{Conclusions}

To the best of our knowledge, results of studies evaluating the effect of the McKenzie method on the change in the sequence of activation of selected muscles during the PHE test have not been published. This study evaluates the level of pain and the change in the sequence of activation of selected muscles during the PHE test in individuals with chronic sacral pain who underwent the McKenzie treatment.

Based on objective research methods, the results of muscle activation in patients with chronic lumbar spine pain significantly differ from the standards set for particular muscles. However, there was an improvement in the muscle activation sequence after the application of a therapeutic procedure based on the McKenzie method, according to tests conducted on the right lower limb. Moreover, the application of the McKenzie method considerably affects the subjective reduction of pain levels.

\section{Acknowledgements}

The authors would like to thank all the participants. Special thanks to mgr Magdalena Lewandowska, the head of the calculation team of Poznań University of Physical Education. This study was funded by the Polish Ministry of Science and Higher Education.

\section{References}

[1] Airaksinen O., Brox J.I., Cedraschi C., Hildebrandt J., Klaber-Moffett J., Kovacs F., Chapter 4 European guidelines for the management of chronic nonspecific low back pain, Eur. Spine J., 2006, 15 (2), 192-300.

[2] BRUno P.A., Bagust J., An investigation into motor pattern differences used during prone hip extension between subjects with and without low back pain, Clin. Chiropr., 2007, June, 10 (2), 68-80.

[3] Bruno P.A., Bagust J., Cook J., Osborne N., An investigation into the activation patterns of back and hip muscles during prone hip extension in non-low back pain subjects: Normal vs. abnormal lumbar spine motion patterns. Clin. Chiropr., 2008, Mar., 11 (1), 4-14.

[4] Bullock-SaXton J.E., Janda V., Bullock M.I., The Influence of Ankle Sprain Injury on Muscle Activation During Hip Extension, Int. J. Sports Med., 1994, 15 (6), 330-334. 
[5] Chance-Larsen K., Littlewood C., Garth A., Prone hip extension with lower abdominal hollowing improves the relative timing of gluteus maximus activation in relation to biceps femoris, Man. Ther., 2010, Feb., 15 (1), 61-65.

[6] Clare H.A., Adams R., MAher C.G., A systematic review of efficacy of McKenzie therapy for spinal pain, Aust. J. Physiother., 2004, 50 (4), 209-216.

[7] COHEN J., Statistical power analysis for a behavioral sciences, Hillsdale, Lawrence Erlbaum Associates, NJ, 1988.

[8] Cram J., Kasman G., Holtz J., Introduction to surface $E M G, 1998$, Aspen Publishing, Gathersburg, Maryland, PA, 336-370, 1.

[9] Danneels L.A., Coorevits P.L., CoOls A.M., Vanderstraeten G.G., CAmBIER D.C., Witvrouw E.E. et al., Differences in electromyographic activity in the multifidus muscle and the iliocostalis lumborum between healthy subjects and patients with sub-acute and chronic low back pain, Eur. Spine J., 2002, Feb., 11 (1), 13-19.

[10] Ferrari S., ManNi T., Bonetti F., Villafañe J.H., Vanti C., A literature review of clinical tests for lumbar instability in low back pain: validity and applicability in clinical practice, Chiropr. Man. Therap., 2015, 23, 14.

[11] Friedli W.G., Hallett M., Simon S.R., Postural adjustments associated with rapid voluntary arm movements 1. Electromyographic data, J. Neurol. Neurosurg. Psychiatry, 1984, 47 (61), 1-622.

[12] Hungerford B., Gilleard W., Hodges P., Evidence of altered lumbopelvic muscle recruitment in the presence of sacroiliac joint pain, Spine (Phila Pa 1976), 2003, Jul., 28 (14), 1593-1600.

[13] Ibrahimaj A., Deliu S., Miftari S., Effectiveness of The McKenzie Method in The Treatment of Low Back Pain in Subacute and Chronic Stage, Research in Physical Education, Sport and Health, 2015, 4 (1), 79-86.

[14] JANDA V., On the Concept of Postural Muscles and Posture in Man, Aust. J. Physiother., 1983, Jun., 29 (3), 83-84.

[15] Kim J.W., Kang M.H., OH J.S., Patients with Low Back Pain Demonstrate Increased Activity of the Posterior Oblique Sling Muscle During Prone Hip Extension, PM R., 2014, May, 6 (5), 400-405.
[16] Lam O.T., Strenger D.M., Chan-Fee M., Pham P.T., Preuss R.A., RobBIns S.M., Effectiveness of the McKenzie Method of Mechanical Diagnosis and Therapy for Treating Low Back Pain: Literature Review With Meta-analysis, J. Orthop. Sports Phys. Ther., 2018, Jun., 48 (6), 476-490.

[17] LEINONEN V., KANKAANPÄÄ M., AIRAKSINEN O., HÄNNINEN O., Back and hip extensor activities during trunk flexion/extension: effects of low back pain and rehabilitation, Arch. Phys. Med. Rehabil., 2000, Jan., 81 (1), 32-37.

[18] Lewis C.L., Sahrmann S.A., Muscle Activation and Movement Patterns During Prone Hip Extension Exercise in Women, J. Athl. Train., 2009, May/Jun., 44 (3), 238-248.

[19] Long A., Donelson R., Fung T., Does it matter which exercise? A randomizes controlled trial of exercise for low back pain, Spine, 2004, Dec. 29 (23), 2593-2602.

[20] McCormack H.M., Horne D.J., Sheather S., Clinical applications of visual analogue scales: a critical review, Psychol. Med., 1988, (18), 1007-1019.

[21] Ogurkowska M.B., Kawalek K., Pathological changes in the lumbar intervertebral discs among professional field hockey players, J. Sports Med. Phys. Fitness, 2016, 56 (1-2), 85-91.

[22] O'Sullivan P.B., Mitchell T., Bulich P., Waller R., HOLTE J., The relationship beween posture and back muscle endurance in industrial workers with flexion-related low back pain, Man. Ther., 2006, Nov., 11 (4), 264-271.

[23] Page P., Frank C., Lardner R., Assessment and Treatment of Muscle Imbalance: The Janda Approach, Chapter 6. Evaluation of movement patterns, 2010, 77-91.

[24] Suehiro T., Mizutani M., Ishida H., Kobara K., Osaka H., WATANABE S., Individuals with chronic low back pain demonstrate delayed onset of the back muscle activity during prone hip extension, J. Electromyogr. Kinesiol., 2015, Aug., 25 (4), 675-680.

[25] Tikkanen O., Haakana P., Pesola A.J., Häkkinen K., RANTALAINEN T. et al., Muscle Activity and Inactivity Periods during Normal Daily Life, PLoS One, 2013, Jan., https://doi.org/ 10.1371/journal.pone.0052228

[28] Vogt L., Pfeifer K., Banzer W., Neuromuscular control of walking with chronic low-back pain, Man. Ther., 2003, Feb., $8(1), 21-28$. 